\title{
Estudo do banco de sementes em povoamentos florestais puros e em uma capoeira de Floresta Ombrófila Aberta, no município de Areia, PB, Brasil
}

\author{
Kilson Pinheiro Lopes ${ }^{1}$, Vênia Camelo de Souza ${ }^{2}$,Leonaldo Alves de Andrade ${ }^{1,3}$ \\ Genaro Viana Dornelas ${ }^{1}$ e Riselane de Lucena Alcântara Bruno ${ }^{1}$
}

Recebido em 4/06/2004. Aceito em 15/07/2005.

\begin{abstract}
RESUMO - (Estudo do banco de sementes em povoamentos florestais puros e em uma capoeira de Floresta Ombrófila Aberta, no município de Areia, PB, Brasil). Em amostragem realizada na estação seca (outubro/2002) em três povoamentos florestais puros constituídos por: sabiá (Mimosa caesalpiniaefolia), sombreiro (Clitoria fairchildiana) e pinus (Pinus sp.) e em uma área em estágio inicial de sucessão ecológica "capoeira", foram coletadas 25 amostras de solos por área em duas profundidades: 0-5 cm e 5-10 cm para determinação da densidade e a composição florística do banco de sementes do solo nas diferentes áreas. A área estudada localiza-se no Balneário Ecológico "O Quebra”, Município de Areia, PB. A maior densidade de sementes ocorreu sempre na profundidade de 0-5 cm, independente do ambiente estudado, havendo, contudo, destaque da área povoada com sombreiro, verificando-se 1.012 e 405 sementes germinadas $/ \mathrm{m}^{2}$ nas profundidades $0-5 \mathrm{~cm}$ e $5-10 \mathrm{~cm}$, respectivamente. A composição florística do banco de sementes foi dominada por espécies da família Urticaceae com mais de $40 \%$ nas duas profundidades estudadas. A forma de vida mais abundante foi a herbácea, independente da profundidade, seguida da arbustiva e arbórea, nos demais ambientes, exceto no sombreiro, onde predominaram arbustos, seguido de ervas e árvores. Excetuando-se o povoamento de sombreiro, os demais ambientes estudados, mesmo apresentando diferentes densidades do banco de sementes do solo, revelaram-se floristicamente similares.
\end{abstract}

Palavras-chave : Silvicultura, sucessão ecológica, regeneração natural

\begin{abstract}
Study of a seed bank in a pure forest population and a ombrophylous secondary forest in Areia Municipal district, PB, Brazil). In sampling accomplished in the drought station of 2002 in three pure forest populations constituted by: sabiá (Mimosa caesalpiniaefolia), sombreiro (Clitoria fairchildiana) and pinus (Pinus sp.) and, an area in initial process of ecological succession, 25 samples of soils were collected by area in two depths: $0-5 \mathrm{~cm}$ and $5-10 \mathrm{~cm}$. The studied universe is located at the Balneary "O Quebra", Areia Municipal district, PB. The biggest seeds density always happened in the 0-5 cm depth, independent of the studied environment. The population of sombreiro showed the biggest stock of seeds, being verified 1,012 and 405 seeds germinated $/ \mathrm{m}^{2}$ in the depths $0-5 \mathrm{~cm}$ and $5-10 \mathrm{~cm}$, respectively. The seeds bank floristic composition was dominated by species of the Urticaceae family with more than $40 \%$ in the two studied depths. The more abundant life form was herbs, independent of the depth, followed by bushes and trees, in all the environments, except in the sombreiro population, where predominated bushes, followed by herbs and trees. Except the sombreiro population, all the studied environments present different densities of the seeds bank of the soil, in spite of being similar in floristic composition.
\end{abstract}

Key words: Forestry, ecological succession, natural regeneration

\section{Introdução}

A recomposição de ecossistemas degradados envolve conhecimentos diversos, principalmente no que se refere à funcionalidade e à dinâmica das espécies, essenciais à formação estrutural das comunidades. $\mathrm{O}$ conhecimento da dinâmica natural e da estrutura do ecossistema são fundamentais no desenvolvimento de modelos de recuperação. $\mathrm{O}$ tipo e a intensidade do distúrbio dependem da natureza do processo de uso da terra que ocorre nas diferentes regiões alteradas, apresentando influência no padrão de recuperação natural do ecossistema (Araújo et al. 2001).

Diversos estudos têm sido realizados com o objetivo de melhor conhecer os processos naturais de recuperação em pastagens, áreas cultivadas para agricultura e florestas alteradas (Uhl et al. 1988; Viana 1990; Wiesenmuller 1995; Vieira, dados não publicados; Nepstad et al. 1998).

O banco de sementes do solo é caracterizado como um depositório de elevada densidade de sementes viáveis e em estado de dormência real ou imposta,

\footnotetext{
1 Universidade Federal da Paraíba, Departamento de Fitotecnia, Centro de Ciências Agrárias, CEP 58397-000, Areia, PB, Brasil

2 Universidade Federal da Paraíba, Departamento de Ciências Básicas e Sociais, Centro de Formação de Tecnólogos, CEP 58220-000, Bananeiras, PB, Brasil

3 Autor para correspondência: landrade@cca.ufpb.br
} 
presentes na superfície ou no interior do solo de determinada área, constituindo-se um sistema dinâmico de entradas e saídas (Hall \& Swaine 1980; Fenner 1985; Kageyama 1987; Garwood 1989; Dalling et al. 1998).

Em regiões tropicais, a regeneração da vegetação, após a retirada da floresta, se dá principalmente por meio da dispersão de sementes oriundas de áreas próximas e do banco de sementes (Uhl et al. 1988; Miriti 1998).

A auto-renovação da floresta é assegurada pela existência do banco de sementes do solo, sendo que a ativação pode proporcionar um aumento de biodiversidade do ecossistema (Oliveira \& Constantin 2001).

Whitmore (1988) relata que o banco de sementes do solo abriga, basicamente, sementes de espécies pioneiras e secundárias iniciais, sendo fator essencial para a regeneração da primeira fase de ocupação e cicatrização de grandes clareiras. Estas espécies representadas por árvores, arbustos e ervas, são comuns como forma de vida predominante do banco de sementes do solo, permanecendo aptas a germinar, por muito tempo, em resposta à alterações da floresta (Bazzaz \& Pickett 1980). As espécies não pioneiras, em geral, germinam logo após dispersadas e vão compor o banco de plântulas, outro componente da regeneração natural.

O conhecimento da distribuição, quantificação e composição populacional do banco de sementes do solo, resulta em valiosa ferramenta para o entendimento da evolução das espécies, permitindo que sejam feitas várias inferências sobre o processo de regeneração natural, assim como a adoção de técnicas de manejo para conservação da diversidade biológica ou recuperação de áreas degradadas (Martins \& Silva 1994; Almeida 2000). Em ecossistemas naturais, o estudo dos bancos de sementes é utilizado para entender e acompanhar os efeitos de interferências humanas, animais ou climáticas no seu equilíbrio (Martins \& Silva 1994), bem como para se compreender como este reage a diferentes culturas, formas de cultivos ou sistemas de uso da terra.

A ativação do banco de sementes do solo se dá após perturbações no ecossistema, seja por uma simples queda de árvores, abertura de uma pequena clareira na floresta ou distúrbios maiores, como abertura de grandes clareiras e desmatamentos. Esses impactos criam condições para que as sementes estocadas entrem em atividade e repovoem a área perturbada (Almeida 2000).

Segundo Campello (1998), uma questão polêmica que envolve técnicos e pesquisadores que trabalham com recuperação de áreas degradadas tem sido a disputa entre função ecológica e origem das espécies vegetais. A razão principal para tal questionamento sobre o uso de espécies exóticas relaciona-se com a possibilidade de uma destas espécies se tornar dominante, interferindo nas fases de sucessão natural, de forma a não permitir o aparecimento de outras plantas ou, em casos extremos, até se tornar uma praga. Contudo, esta possibilidade também existe com espécies nativas, além do fato de que grande número de espécies introduzidas já ter sido testado, sem expressar características ambientais inadequadas. Odum (1988) enfatiza que, a princípio os padrões de sucessão mostram-se mais dependentes da função ecológica que cada indivíduo compõe no sistema do que da identidade botânica e origem da espécie.

Devido à importância e a carência de informações básicas sobre a dinâmica da regeneração natural em povoamentos homogêneos, este trabalho objetivou avaliar qualitativa e quantitativamente o banco de sementes em sub-bosques de povoamentos florestais e em uma comunidade autóctone em estágio inicial de sucessão ecológica.

\section{Material e métodos}

Área de estudo - Está localizada no Balneário Ecológico "O Quebra", no Município de Areia, região do brejo Paraíbano (658'12"S e 3542'14”W), envolvendo talhões de três povoamentos florestais puros com sabiá, sombreiro e pinus, com idade média de 15 anos e uma área em estágio de sucessão ecológica inicial (capoeira). As áreas apresentam em torno de 2 hectares cada uma, e foram escolhidas por serem representativas das áreas de reflorestamento da região. O referido balneário está inserido na zona urbana de Areia, PB, sendo que a frente norte é limitada por pastagem. $\mathrm{O}$ fragmento de floresta nativa mais próximo dos povoamentos está situado acerca de $2 \mathrm{~km}$.

De acordo com Brasil (1972), o clima da região é classificado como As (quente e úmido), de acordo com a classificação de Köppen, correspondendo a ambiente úmido com precipitação média anual próxima de $1.500 \mathrm{~mm} / \mathrm{ano}$ e a umidade relativa do ar mantém-se em torno de 75 a $87 \%$. A temperatura média anual oscila entre 22 a $26^{\circ} \mathrm{C}$.

Caracterização do banco de sementes do solo - Para estudo da densidade e composição florística do banco de sementes, foram coletadas amostras, no mês de outubro, que corresponde à estação seca na região, realizadas de forma aleatória, em número de 25 
amostras no interior de cada ambiente estudado. Para isso foram utilizados gabaritos de madeira, com a dimensão $0,23 \times 0,17 \mathrm{~m}\left(0,0391 \mathrm{~m}^{2}\right)$ que, colocados sobre a superfície do solo, permitiram a padronização das amostras coletadas em duas profundidades: 0 a $5 \mathrm{~cm}$ e 5 a $10 \mathrm{~cm}$.

As amostras coletadas foram armazenadas em sacos plásticos e transportadas à unidade de germinação (casa de vegetação) no Centro de Ciências Agrárias da Universidade Federal da Paraíba, no município de Areia, onde o material foi distribuído aleatoriamente em bandejas de alumínio $(0,23 \mathrm{~m} \times$ $0,17 \mathrm{~m} \times 0,05 \mathrm{~m})$, de forma a não favorecer amostras, ambiente ou profundidade de coleta.

A casa de vegetação apresentava-se revestida lateralmente com sombrite, que permite $50 \%$ de sombreamento e coberta com plástico transparente, $o$ que a mantinha isolada e protegida contra a contaminação de propágulos externos ao ambiente e impactos da chuva.

O estudo foi monitorado com irrigações feitas de acordo com a necessidade e durante todo o período do trabalho.

Avaliação do banco de sementes do solo - O experimento foi conduzido durante três meses, período em que houve emergência de plântulas e as contagens e identificações realizadas semanalmente.

Empregou-se o delineamento experimental inteiramente casualizado em esquema fatorial representado por quatro ambientes e duas profundidades, com 25 repetições. O número de sementes germinadas, identificadas em cada semana, foi logaritimizado e submetido a testes de normalidade "Teste de Lilliefors" (Campos 1979) e homogeneidade da variância "Teste de Bartlett" (Zar 1996). Finalmente o número de sementes germinadas foi analisado pelo método de estatística paramétrica, com o uso de análise de variância simples e comparação das médias de densidade do banco de sementes pelo teste de Tukey (Warr et al. 1993).

Os indivíduos identificados foram classificados por forma de vida em: arbórea (vegetal lenhoso com altura igual ou maior do que $5 \mathrm{~m}$ ), arbustiva (vegetal lenhoso menor que $5 \mathrm{~m}$ de altura, ramificando-se a partir da base) e herbácea (vegetal não lignificado, incluindo gramíneas e ciperáceas). No caso de não identificação, ou incerteza de alguma plântula, esta era transplantada para saco de polietileno e cultivada em viveiro para posterior identificação. A identificação e a classificação foram feitas pela equipe técnica do Laboratório de Botânica do CCA/UFPB.
Com o intuito de fornecer maiores informações foi calculado o índice de similaridade de Sorensen $\left(\mathrm{CC}_{\mathrm{s}}\right)$ para comparar a composição florística no banco de sementes do solo dos diferentes ambientes estudados (Brower e Zar 1984).

$$
C C s=\frac{2 . c}{S 1+S 2}
$$

Considerando que:

$c=$ número de espécies comuns nas duas comunidades

$S 1=$ número de espécies da comunidade $\mathrm{A}$

$S 2$ = número de espécies da comunidade $\mathrm{B}$

Amostras de solos foram coletadas e encaminhadas para o Laboratório de Análise de Solos do Centro de Ciências Agrárias da Universidade Federal da Paraíba em Areia, PB, Brasil, com o intuito de se avaliar a qualidade química e de fertilidade do solo das áreas estudadas.

\section{Resultados e discussão}

A maior densidade de sementes viáveis ocorreu sempre na profundidade de $0-5 \mathrm{~cm}$, independente do ambiente estudado, superando a profundidade de $5-10 \mathrm{~cm}$, com mais que o dobro do número de sementes germinadas $/ \mathrm{m}^{2}$. No ambiente compreendido pelo povoamento de sombreiro, verificou-se 1.012 e 405 sementes germinadas $/ \mathrm{m}^{2}$ nas profundidades $0-5 \mathrm{~cm} \mathrm{e}$ 5-10 cm, respectivamente, superando os demais ambientes (Tab. 6). Dados semelhantes foram obtidos por Araújo et al. (2001), em florestas sucessionais de 17 anos, relatando ainda que tais valores estão incluídos no limite para banco de sementes de florestas tropicais em sucessão. Whitmore (1983) afirma que a maioria das sementes de pioneiras capazes de germinar, em florestas tropicais, encontra-se nos primeiros $5,0 \mathrm{~cm}$ de profundidade do solo superficial.

A maior proporção de sementes germinadas ocorreu da segunda à quarta semana de observação, onde foi constatada, nos demais ambientes estudados, emergência em torno de $20 \%$ e $30 \%$, nas profundidades de $0-5 \mathrm{~cm}$ e $5-10 \mathrm{~cm}$, respectivamente, com pequenas oscilações nas semanas seguintes, que variaram de $8 \%$ a $5 \%$ e decréscimo nas duas últimas semanas (Fig. 1). É possível que a maior proporção de sementes germinadas, nas primeiras quatro semanas, nas diferentes camadas do solo seja consequiência da superação da dormência após exposição à luz e maior amplitude de temperatura, de modo semelhante ao verificado por Baskin \& Baskin (1998) e Costa \& 


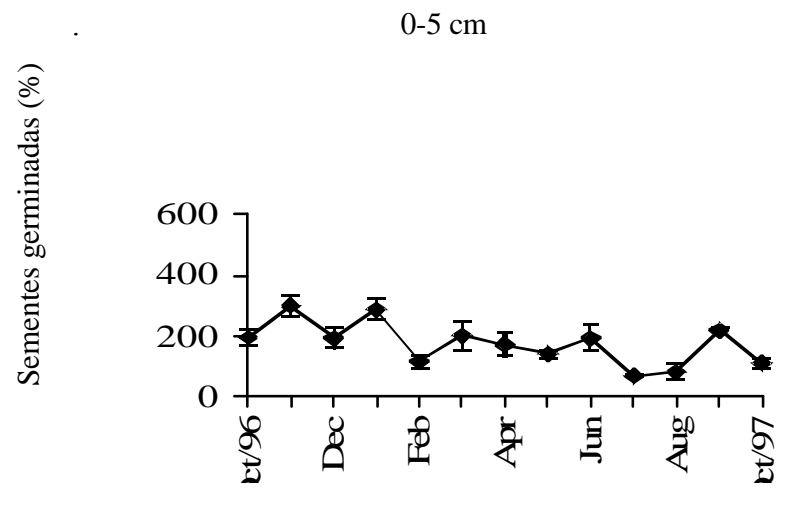

$5-10 \mathrm{~cm}$

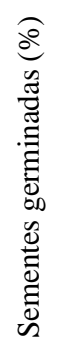

Araújo (2003). Estas modificações das condições ambientais, contrárias àquelas estabelecidas sob o dossel da floresta, são também enfatizadas por Bazzaz \& Pickett (1980) e Whitmore (1990). Os resultados apresentados evidenciam a importância potencial do banco de sementes presente no solo, na capacidade de regeneração de áreas após alguma perturbação.

O banco de sementes do solo, correspondente ao povoamento de sombreiro apresentou a maior riqueza florística, com 38 espécies, enquanto que nos outros dois povoamento, sabiá e pinus, constatou-se 24 e 18 espécies, respectivamente, já na área em estágio de sucessão ecológica "capoeira", registrou-se 20 espécies (Tab. 1, 2, 3 e 4). Estes resultados concordam com os obtidos por Campello (1998) onde, por meio de avaliação da sucessão em reflorestamento com 12 anos, em áreas de solo arenoso degradado em Porto Trombetas, PA, verificou que, sob as parcelas de uma leguminosa fixadora exótica (Acacia mangium), uma leguminosa fixadora nativa (Sclerobium paniculatum), duas mirtáceas exóticas (Eucalyptus pellita e Eucalyptus citriodora) e uma celastrácea nativa (Goupia glabra), a maior riqueza de espécies nativas oriundas da regeneração natural e a maior biomassa vegetal podem ser encontradas sob os reflorestamentos com leguminosas fixadoras de nitrogênio.

Figura 1. Percentagem de sementes germinadas em $0,9775 \mathrm{~m}^{2}$, durante 12 semanas, nos povoamentos e respectivas profundidades estudadas, na região do brejo no município de Areia, PB, Brasil.

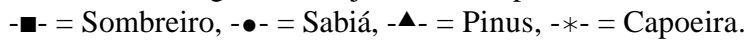

Tabela 1. Lista das espécies e respectivas formas de vida no povoamento de sombreiro, onde: forma de vida (FV) e número de sementes (NS) nas profundidades $\mathrm{P}_{1}(0-5 \mathrm{~cm})$ e $\mathrm{P}_{2}(5-10 \mathrm{~cm})$ foram identificadas em $0,9775 \mathrm{~m}^{2}$, no banco de sementes no povoamento, na região do brejo, no município de Areia, PB, Brasil.

\begin{tabular}{|c|c|c|c|c|c|}
\hline \multirow[t]{2}{*}{ Nome científico } & \multirow[t]{2}{*}{ Família } & \multirow[t]{2}{*}{ Nome vulgar } & \multirow[t]{2}{*}{ FV } & \multicolumn{2}{|c|}{ NS } \\
\hline & & & & $P_{1}$ & $\mathrm{P}_{2}$ \\
\hline Ageratum conyzoides $\mathrm{L}$. & Asteraceae & mentrasto & erva & 3 & 1 \\
\hline Alocasia macrorhizas Schott. & Araceae & taioba & arbusto & 1 & 1 \\
\hline Alternanthera tenella Colla & Amaranthaceae & quebra-panela & erva & 5 & - \\
\hline Amaranthus deflexus L. & Amaranthaceae & bredo de porco & erva & 6 & 1 \\
\hline Bidens pilosa $\mathrm{L}$. & Compositae & picão preto & arbusto & 2 & 1 \\
\hline Boerhaavia diffusa Engelm. \& A. Gray. & Nyctaginaceae & pega pinto & erva & 5 & 1 \\
\hline Canna indica $\mathrm{L}$. & Cannaceae & biri & erva & - & 1 \\
\hline Cestrum laevigatum Schlecht. & Solanaceae & maria branca & arbusto & 7 & - \\
\hline Chamaesyce hyssopifolia Small. & Euphorbiaceae & leiteiro & arbusto & 2 & - \\
\hline Clitoria fairchildiana Howard. & Leguminosae & sombreiro & árvore & 5 & - \\
\hline Commelina sp. & Commelinaceae & trapoeraba & erva & 9 & 15 \\
\hline Cyperus ferax Benth. & Cyperaceae & estrela-de-são-joão & erva & 75 & 28 \\
\hline Digitaria horizontalis Willd. & Gramineae & capim-de-roça & erva & 18 & 20 \\
\hline Emilia sonchifobia Benth. & Asteraceae & emilia & erva & 4 & 4 \\
\hline Hydrocotyles $\mathrm{sp}$ & Apiaceae & orelha-de-onça & erva & 108 & 9 \\
\hline Impatiens balsamina Hook.f. & Balsaminaceae & beijo & erva & 86 & 11 \\
\hline Ipomoea ararifolia (Ders.) Roem \& Schult. & Convolvulaceae & salsa & erva & - & 1 \\
\hline Momordica charantia Descourt. & Cucurbitaceae & melão de são-caetano & erva & 16 & $\begin{array}{c}1 \\
\text { continua }\end{array}$ \\
\hline
\end{tabular}


Tabela 1 (continuação)

\begin{tabular}{|c|c|c|c|c|c|}
\hline \multirow[t]{2}{*}{ Nome científico } & \multirow[t]{2}{*}{ Família } & \multirow[t]{2}{*}{ Nome vulgar } & \multirow[t]{2}{*}{ FV } & \multicolumn{2}{|c|}{ NS } \\
\hline & & & & $\mathrm{P}_{1}$ & $\mathrm{P}_{2}$ \\
\hline Nicotiana tabacum L. & Solanaceae & fumo & erva & - & 2 \\
\hline Oxalis sp. & Oxalidaceae & trevo & erva & 2 & 1 \\
\hline Panicum maximum Hochst. ex A. Rich. & Gramineae & capim-colonial & erva & 3 & 4 \\
\hline Phylantus niruri Thunb. & Euphorbiaceae & quebra-pedras & erva & 16 & 6 \\
\hline Physalis sp. & Solanaceae & camapu & arbusto & 7 & 9 \\
\hline Pilea sp. & Urticaceae & brilhantina & erva & 3 & 1 \\
\hline Polygonum hydropiperoides Michx. & Polygonaceae & pimenta-d'água & arbusto & 4 & 7 \\
\hline Portulaca oleracea L. & Portulacaceae & breldroega & erva & 3 & 2 \\
\hline Raphanus raphanistrum Cav. & Cruciferaceae & nabiça & arbusto & 1 & - \\
\hline Richardia grandiflora Britton. & Rubiaceae & vassourão & erva & 2 & 5 \\
\hline Ricinus comunis L. & Euphorbiaceae & mamona & arbusto & 1 & - \\
\hline Solanum capsicoides Hort. Par. ex Lam. & Solanaceae & gogoia & erva & 1 & - \\
\hline Turnera sp. & Turneraceae & diadema & erva & - & 1 \\
\hline Verbesina diversifolia DC. & Asteraceae & camará-de-bucha & erva & 1 & 1 \\
\hline sp. 1 & Caryophillaceae & borragem & erva & 1 & - \\
\hline sp. 2 & Polypodiaceae & samambaia & erva & 8 & 1 \\
\hline sp. 3 & Labiatae & labiata & arbusto & 1 & - \\
\hline sp. 4 & Asteraceae & não identificada & erva & 30 & 11 \\
\hline sp. 5 & Urticaceae & não identificada & arbusto & 495 & 167 \\
\hline sp. 6 & Euphorbiaceae & não identificada & arbusto & 43 & 23 \\
\hline
\end{tabular}

Tabela 2. Lista das espécies e respectivas formas de vida no povoamento de sabiá, onde: forma de vida (FV) e número de sementes (NS) nas profundidades $\mathrm{P}_{1}(0-5 \mathrm{~cm})$ e $\mathrm{P}_{2}(5-10 \mathrm{~cm})$ foram identificadas em $0,9775 \mathrm{~m}^{2}$, no banco de sementes no povoamento, na região do brejo, no município de Areia, PB, Brasil.

\begin{tabular}{|c|c|c|c|c|c|}
\hline \multirow[t]{2}{*}{ Nome científico } & \multirow[t]{2}{*}{ Família } & \multirow[t]{2}{*}{ Nome vulgar } & \multirow[t]{2}{*}{$\mathrm{FV}$} & \multicolumn{2}{|c|}{ NS } \\
\hline & & & & $\mathrm{P}_{1}$ & $\mathrm{P}_{2}$ \\
\hline Ageratum conyzoides $\mathrm{L}$. & Asteraceae & mentrasto & Erva & 16 & 1 \\
\hline Amaranthus deflexus L. & Amaranthaceae & bredo-de-porco & erva & 2 & - \\
\hline Bidens pilosa $\mathrm{L}$. & Compositae & picão-preto & arbusto & 1 & 2 \\
\hline Cecropia sp. & Cecropiaceae & imbaúba & árvore & 3 & - \\
\hline Cleome spinosa Jacq. & Capparaceae & muçambê & arbusto & 1 & - \\
\hline Commelina sp. & Commelinaceae & trapoeraba & erva & 31 & 15 \\
\hline Conyza sp. & Asteraceae & rabo-de-rapousa & erva & 1 & - \\
\hline Croton lobatus Forsk. & Euphorbiaceae & café-bravo & erva & 1 & - \\
\hline Cyperus ferax Benth. & Cyperaceae & estrela-de-são-joão & erva & 10 & 2 \\
\hline Digitaria horizontalis Willd. & Gramineae & capim-de-roça & erva & 29 & 18 \\
\hline Emilia sonchifobia Benth. & Asteraceae & emilia & erva & 3 & - \\
\hline Melochia sp. & Esterculiaceae & relógio & arbusto & 1 & - \\
\hline Merremia aegypytia Gamble. & Convolvulaceae & jitirana & erva & 2 & - \\
\hline Mimosa caesalpiniaefolia Benth. & Leguminosae & sabiá & árvore & 24 & - \\
\hline Panicum maximum Hochst. ex. A. Rich. & Gramineae & capim-colonial & erva & 88 & 10 \\
\hline Physalis sp. & Solanaceae & camapu & arbusto & 3 & - \\
\hline Ricardia grandiflora Britton. & Rubiaceae & vassourão & erva & 26 & 7 \\
\hline Turnera sp. & Turneraceae & diadema & erva & 1 & 1 \\
\hline Vernonia scabra K. Schum. & Compositae & manjericão-de-cavalo & arbusto & 1 & - \\
\hline sp. 1 & Polypodiaceae & samambaia & erva & 2 & - \\
\hline sp. 3 & Urticaceae & não identificada & arbusto & 35 & 6 \\
\hline sp. 4 & Rubiaceae & não identificada & erva & 6 & - \\
\hline sp. 5 & Labiatae & não identificada & arbusto & 2 & - \\
\hline sp. 2 & Asteraceae & não identificada & erva & 90 & 20 \\
\hline
\end{tabular}


Tabela 3. Lista das espécies e respectivas formas de vida no povoamento de pinus, onde: forma de vida (FV) e número de sementes (NS) nas profundidades $\mathrm{P}_{1}(0-5 \mathrm{~cm})$ e $\mathrm{P}_{2}(5-10 \mathrm{~cm})$ foram identificadas em $0,9775 \mathrm{~m}^{2}$, no banco de sementes do povoamento, na região do brejo, no município de Areia, PB, Brasil.

\begin{tabular}{|c|c|c|c|c|c|}
\hline \multirow[t]{2}{*}{ Nome científico } & \multirow[t]{2}{*}{ Família } & \multirow[t]{2}{*}{ Nome vulgar } & \multirow[t]{2}{*}{$\mathrm{FV}$} & \multicolumn{2}{|c|}{ NS } \\
\hline & & & & $\mathrm{P}_{1}$ & $\mathrm{P}$ \\
\hline Acanthospermum hispidum DC. & Compositae & carrapicho-de-cigano & erva & 1 & - \\
\hline Ageratum conyzoides L. & Asteraceae & mentrasto & erva & - & 1 \\
\hline Bidens pilosa $\mathrm{L}$. & Asteraceae & picão-preto & arbusto & 1 & 6 \\
\hline Cestrum laevigatum Schlecht. & Solanaceae & maria-branca & arbusto & 1 & - \\
\hline Commelina sp. & Commelinaceae & trapoeraba & erva & 43 & 7 \\
\hline Croton lobatus Forsk. & Euphorbiaceae & café-bravo & erva & 1 & - \\
\hline Cyperus ferax Benth. & Cyperaceae & estrela-de-são-joão & erva & 13 & 13 \\
\hline Digitaria horizontalis Willd. & Gramineae & capim-de-roça & erva & 19 & 18 \\
\hline Emilia sonchifobia Benth. & Asteraceae & emilia & erva & 3 & - \\
\hline Mimosa caesalpiniaefolia Benth. & Leguminosae & sabiá & árvore & - & 1 \\
\hline Panicum maximum Hochst. ex. A. Rich. & Gramineae & capim-colonial & erva & 2 & 6 \\
\hline Physalis sp. & Solanaceae & camapu & arbusto & 2 & 13 \\
\hline Polygonum hydropiperoides Michx. & Polygonaceae & pimenta-d'água & arbusto & 3 & 5 \\
\hline Richardia grandiflora Britton. & Rubiaceae & vassourão & erva & 3 & 4 \\
\hline Sida sp. & Malvaceae & relógio & arbusto & 1 & - \\
\hline Triumfetta sp. & Tiliaceae & carrapichinho & erva & 1 & - \\
\hline sp. 1 & Asteraceae & não identificada & erva & 6 & 9 \\
\hline sp. 2 & Urticaceae & não identificada & arbusto & 43 & 10 \\
\hline
\end{tabular}

Tabela 4. Lista das espécies e respectivas formas de vida em área de capoeira, onde: forma de vida (FV) e número de sementes (NS) nas profundidades $\mathrm{P}_{1}(0-5 \mathrm{~cm})$ e $\mathrm{P}_{2}(5-10 \mathrm{~cm})$ foram identificadas em $0,9775 \mathrm{~m}^{2}$, no banco de sementes da área, na região do brejo no município de Areia, PB, Brasil.

\begin{tabular}{|c|c|c|c|c|c|}
\hline \multirow[t]{2}{*}{ Nome científico } & \multirow[t]{2}{*}{ Família } & \multirow[t]{2}{*}{ Nome vulgar } & \multirow[t]{2}{*}{$\mathrm{FV}$} & \multicolumn{2}{|c|}{ NS } \\
\hline & & & & $\mathrm{P}_{1}$ & $\mathrm{P}_{2}$ \\
\hline Ageratum conyzoides $\mathrm{L}$. & Asteraceae & mentrasto & erva & 51 & 25 \\
\hline Alternanthera tenella Colla & Amaranthaceae & quebra-panela & arbusto & 7 & - \\
\hline Bidens pilosa $\mathrm{L}$. & Asteraceae & picão-preto & arbusto & 2 & - \\
\hline Centrosema pubescens Benth. & Leguminosae & espia-caminho & erva & 2 & - \\
\hline Cestrum laevigatum Schlecht. & Solanaceae & maria-branca & arbusto & 9 & 1 \\
\hline Commelina sp. & Commelinaceae & trapoeraba & erva & 47 & 45 \\
\hline Cyperus ferax Benth. & Cyperaceae & estrela-de-são-joão & erva & 2 & - \\
\hline Digitaria horizontalis Willd. & Gramineae & capim-de-roça & erva & 58 & 31 \\
\hline Emilia sonchifobia Benth. & Asteraceae & emilia & erva & 1 & - \\
\hline Leucaena leucocephala de Wit. & Leguminosae & leucena & árvore & 12 & 1 \\
\hline Merremia aegypta Gamble. & Convolvulaceae & jitirana & erva & 5 & - \\
\hline Panicum maximum Hochst. ex. A. Rich. & Gramineae & capim-colonial & erva & 40 & 11 \\
\hline Physalis sp. & Solanaceae & camapu & arbusto & 10 & 1 \\
\hline Porophyllum ruderale Cars. & Asteraceae & cravo-de-urubu & arbusto & 1 & - \\
\hline Ricardia grandiflora Britton. & Rubiaceae & vassourão & erva & 9 & - \\
\hline Spermacoce verticillata $\mathrm{L}$. & Rubiaceae & vassoura-de-botão & erva & 1 & 2 \\
\hline Stylosanthes guianenses Sw. & Fabaceae & stilosante & arbusto & - & 1 \\
\hline Turnera sp. & Turneraceae & diadema & erva & - & 1 \\
\hline sp. 1 & Asteracee & não identificada & erva & 54 & 11 \\
\hline sp. 2 & Rubiaceae & não identificada & erva & 2 & - \\
\hline
\end{tabular}


A forma de vida que predominou na maioria das áreas estudadas, independente da profundidade, foi a herbácea, seguida da arbustiva e arbórea, exceto na área compreendida pelo sombreiro, em ambas profundidades, e na área compreendida pelo sabiá na profundidade de $0-5 \mathrm{~cm}$, onde houve predominância de arbustos, seguido de ervas e árvores (Fig. 2). Segundo Uhl \& Clark (1983), a adjacência de florestas em sucessão avançada, justifica a tendência obtida para forma de vida, considerando que um dos fatores que influencia na recuperação da composição de espécies é a fonte de sementes. Contudo, a predominância de uma determinada forma de vida num ambiente depende, principalmente, do tipo de pressão sofrida, não somente na área, mas na microrregião, uma vez que em áreas
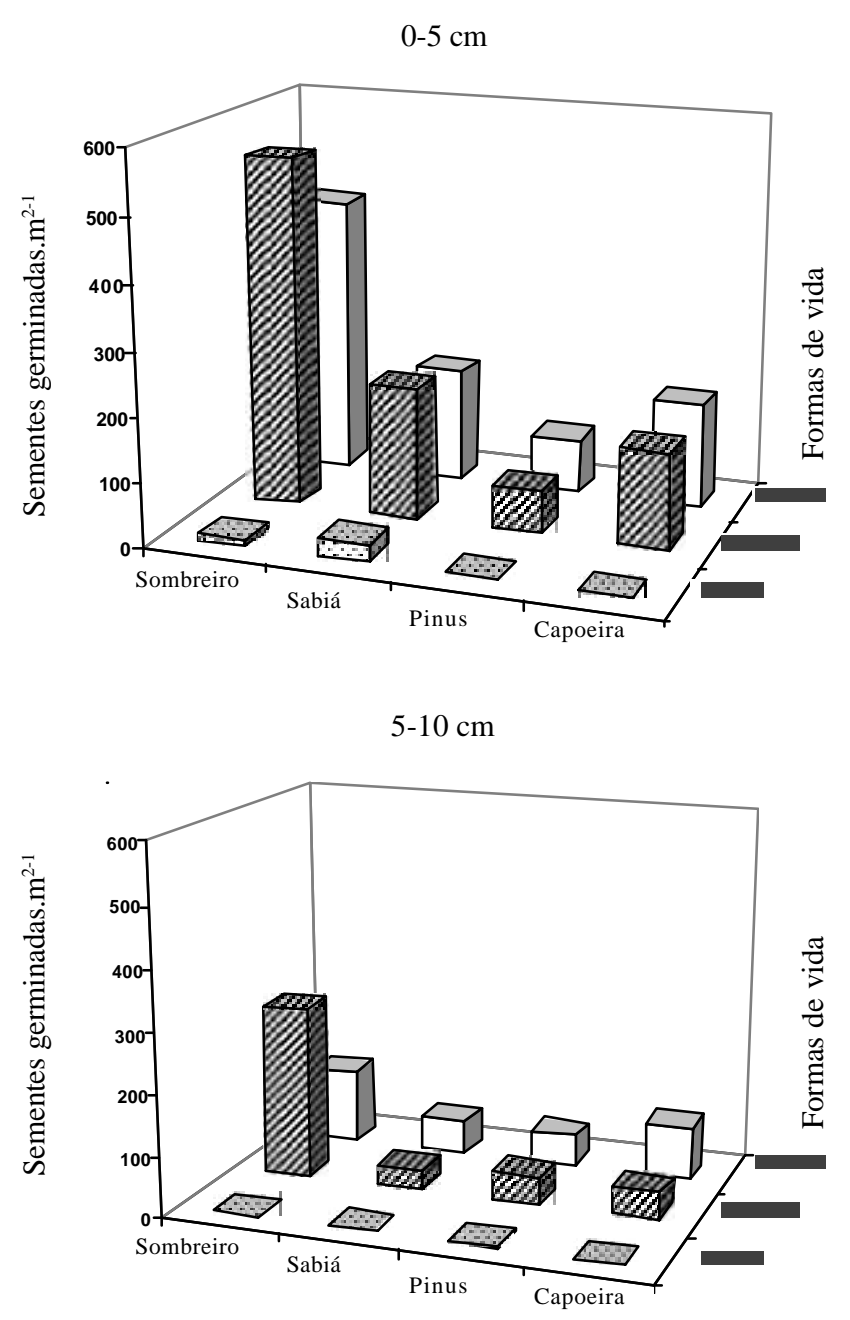

Povoamentos

Figura 2. Densidade em $\mathrm{m}^{2}$ do banco de sementes em duas profundidades do solo de acordo com a forma de vida, nos diferentes povoamentos da região do brejo, no município de Areia, PB, Brasil. onde existiu a degradação do ecossistema florestal para a introdução de pastagens ou para cultivo, há um domínio de espécies invasoras herbáceas, gramíneas e arbustos (Araújo et al. 2001).

Em relação à composição florística na profundidade de 0 a $5 \mathrm{~cm}$, as espécies que ocorreram em maior densidade foram uma espécie da família Urticaceae (não identificada), na área do povoamento de sombreiro com 48,91\%; uma espécie da família Asteraceae (não identificada) e o Panicum maximum (capim-colonião), no povoamento de sabiá com $21 \%$ cada; a Urticaceae e a Commelina sp. (trapoeraba), no povoamento de pinus, cada um com $28 \%$; e na área de capoeira ocorreram as espécies Digitaria horizontalis (capim-de-roça) com 18\%, uma espécie da família Asteraceae (não identificada) com 17\%, o Eupatorium levigatum (mentrasto) e o Panicum maximum (capim-colonião) cada um com $15 \%$. Já na profundidade de $5-10 \mathrm{~cm}$ uma espécie de Urticaceae (não identificada) ocorreu no povoamento de sombreiro com 41\%; no povoamento de pinus registrou-se uma espécie da família Asteraceae (não identificada) e a Digitaria horizontalis (capim-de-roça) com $25 \%$ e $23 \%$, respectivamente; esta última ocorreu também no povoamento de pinus com $19 \%$, enquanto que na capoeira constatou-se a ocorrência da Commelina sp. (trapoeraba) e da Digitaria horizontalis (capim-deroça), com $34 \%$ e $23 \%$, respectivamente. Herrera et al. (1993) enfatizam que na sucessão primária, processos previstos para situações onde ocorre a degradação do solo, as espécies pioneiras de outras áreas contíguas, mas anteriormente inexistentes ou com baixa densidade, podem ser introduzidas pelos agentes dispersores naturais, direcionando dessa maneira, a sucessão para a formação de um ambiente diferente daquele que existia.

De acordo com a análise de química e fertilidade dos solos nos ambientes estudados (Tab. 5), observa-se que a maior quantidade de carbono e matéria orgânica foi constatada no solo da área de capoeira, superando as duas áreas que compreendiam as populações de leguminosas arbóreas fixadoras de nitrogênio, ou seja, a de sombreiro e a de sabiá. Estes resultados fogem do que se espera em áreas com leguminosas arbóreas, tidas como ótimas fornecedoras de cobertura vegetal, conjugada com efeitos de maior duração, como oferta contínua de nitrogênio, elevada deposição de matéria orgânica de rápida decomposição, entre outros fatores, que atuam como ativadores e reguladores dos recursos disponíveis, de forma, a permitir o surgimento de espécies mais exigentes (Campello 1998). Segundo 
Tabela 5. Análise química e de fertilidade dos solos nos povoamentos em que foi realizado o estudo do banco de sementes do solo, Areia, PB, Brasil.

\begin{tabular}{|c|c|c|c|c|c|}
\hline \multirow[t]{2}{*}{ Área } & \multirow{2}{*}{$\begin{array}{c}\mathrm{pH} \\
(1: 2,5)\end{array}$} & $\mathrm{P}$ & $\mathrm{K}$ & $\mathrm{C}$ & MO \\
\hline & & \multicolumn{2}{|c|}{$\mathrm{mg} \cdot \mathrm{dm}^{-3}$} & \multicolumn{2}{|c|}{ g.kg ${ }^{1}$} \\
\hline Sombreiro & 6,0 & 33,24 & 115,13 & 16,04 & 27,65 \\
\hline Sabiá & 4,8 & 2,90 & 69,09 & 16,04 & 27,65 \\
\hline Pinus & 4,9 & 7,73 & 59,89 & 16,57 & 28,57 \\
\hline Capoeira & 4,9 & 2,21 & 50,69 & 18,24 & 31,44 \\
\hline Área & $\mathrm{Na}$ & $\mathrm{Ca}$ & $\begin{array}{c}\mathrm{Mg} \\
\mathrm{Cmol}^{-\mathrm{dm}^{-3}}\end{array}$ & Al & $\mathrm{H}+\mathrm{Al}$ \\
\hline Sombreiro & 0,07 & 5,80 & 0,70 & 0,00 & 3,55 \\
\hline Sabiá & 0,03 & 1,13 & 0,77 & 1,05 & 10,97 \\
\hline Pinus & 0,07 & 1,61 & 0,59 & 0,85 & 8,91 \\
\hline Capoeira & 0,07 & 0,71 & 0,86 & 1,10 & 10,15 \\
\hline
\end{tabular}

Sprent \& Sutherland (1990) há vários fatores do solo e do ambiente que podem interferir na capacidade de nodulação e fixação de $\mathrm{N}_{2}$ do ar pelas leguminosas arbóreas. Contudo, na área de capoeira, foi constatada a presença de leguminosas fixadoras de nitrogênio, como a Centrosema pubescens e Leucaena leucocephala, apesar de em menor freqüência (Tab. 4). O solo da área povoada com sombreiro apresentou os maiores valores de $\mathrm{P}, \mathrm{K}$ e $\mathrm{Ca}$ e um $\mathrm{pH}$ 6,0 , mais próximo da neutralidade, quando comparado aos solos das demais áreas estudadas, que apresentaram $\mathrm{pH}$ ácido, em torno de 4,8 e maior quantidade de Al (Tab. 5). Segundo Campello (1998), o sombreiro é recomendado para plantios em solos ácidos e áreas com problemas de drenagem, o que pôde ter contribuído, no presente estudo, com o melhor pH e maiores valores de $\mathrm{K}$ e $\mathrm{Ca}$, do solo na área do povoamento com essa espécie.

O povoamento de sombreiro evidenciou índices de similaridade muito próximos, no banco de sementes,

Tabela 6. Densidade de sementes nos diferentes povoamentos e profundidades estudados.

\begin{tabular}{lcr}
\hline & \multicolumn{2}{c}{ Sementes germinadas $/ \mathrm{m}^{2}$} \\
\cline { 2 - 3 } Povoamentos & $0-5 \mathrm{~cm}$ & \multicolumn{1}{c}{$5-10 \mathrm{~cm}$} \\
\hline Sombreiro & $1012,79 \pm 126,39 \mathrm{aA}$ & $405,12 \pm 67,63 \mathrm{aB}$ \\
Sabiá & $415,35 \pm 70,93 \mathrm{bA}$ & $79,80 \pm 20,58 \mathrm{bB}$ \\
Pinus & $152,43 \pm 35,61 \mathrm{cA}$ & $96,16 \pm 23,64 \mathrm{bB}$ \\
Capoeira & $319,18 \pm 51,77 \mathrm{bA}$ & $134,02 \pm 24,50 \mathrm{bB}$ \\
\hline
\end{tabular}

Médias seguidas pela mesma letra minúscula nas colunas e maiúsculas nas linhas não diferem entre si pelo teste de Tukey a $5 \%$ de probabilidade. quando comparado aos dos povoamentos de sabiá $(0,48)$, pinus $(0,46)$ e à área de capoeira $(0,41)$ (Tab. 7). Estes resultados podem ter ocorrido devido a proximidade das áreas, possibilitando uma dispersão mais efetiva, ou mesmo, pelo fato do povoamento de sombreiro, por suas características físicas, facilitar ou favorecer a entrada de um maior número de sementes de espécies secundárias. Os menores índices de similaridade $(\cong 0,30)$, ocorreram entre bancos de sementes dos povoamentos de pinus, sabiá e da área de capoeira. Segundo Hall \& Swane (1980) a similaridade entre bancos de sementes, principalmente restritos a uma área ou região, é relativamente elevada e, geralmente, maior do que entre vegetações.

Tabela 7. Índice de similaridade de Sorensen, entre bancos de sementes dos povoamentos estudados, na região do brejo, no município de Areia, PB, Brasil.

\begin{tabular}{lcccc}
\hline & Sombreiro & Sabiá & Pínus & Capoeira \\
\hline Sombreiro & - & - & - & - \\
Sabiá & 0,48 & - & - & - \\
Pinus & 0,46 & 0,31 & - & - \\
Capoeira & 0,41 & 0,30 & 0,29 & - \\
\hline
\end{tabular}

Os resultados obtidos nos levam a concluir que a maior densidade de sementes no solo ocorreu na profundidade de $0-5 \mathrm{~cm}$, sendo o povoamento de sombreiro o responsável pelo maior número de sementes, principalmente, de espécies pioneiras, típicas formadoras de banco de sementes persistentes.

A forma de vida herbácea foi a mais abundante, independente da profundidade, seguida da arbustiva e da arbórea, nos demais ambientes, exceto no sombreiro, onde predominaram arbustos, seguidos de ervas e árvores.

A família Urticaceae predominou no banco de sementes do solo, com mais de $40 \%$, no povoamento florestal puro com sombreiro, nas duas profundidades do solo, juntamente com outras espécies que ocorreram nos demais povoamentos, caracterizadas colonizadoras imediatas no caso de alteração da floresta, como: espécies da família Asteraceae e Gramineae, a Commelina sp. e Ageratum conyzoides.

Com exceção do povoamento florestal puro com sombreiro, os demais ambientes estudados, apesar de apresentarem diferentes densidades do banco de sementes do solo, apresentaram composição florística de espécies similares, capazes de germinar e emergir logo após alteração do ambiente sob o dossel. 


\section{Referências bibliográficas}

Almeida, D.S. 2000. Recuperação ambiental da mata atlântica. Ilhéus, Editus.

Araujo, M.M.; Oliveira, F.A.; Vieira, I.C.G.; Barros, P.L.C. \& Lima, C.A.T. 2001. Densidade e composição florística do banco de sementes do solo de florestas sucessionais na região do Baixo Rio Guamá, Amazônia Oriental. Scientia Forestalis 59: 115-130.

Baskin, C.C. \& Baskin, J.M. 1998. Seeds, ecology, biogeography, and evolution of dormancy and germination. New York, Academic Press.

Bazzaz, F.A. \& Pickett, S.T.A. 1980. Physiological ecology of tropical succession: a comparativereview. Annual review on ecology and sistematics 11: 287-310.

Brasil, Ministério da Agricultura. 1972. Levantamento exploratório, reconhecimento de solos do Estado da Paraíba. Rio de Janeiro. MA/Conta/Usaid/Sudene, 670p. (Boletim Técnico,15).

Brower, J.E. \& ZAR, J.H. 1984. Field and laboratory methods for general ecology. Lowa, Brown Publishers.

Campello, E.F.C. 1998. Sucessão vegetal na recuperação de áreas degradadas. Pp.183-196. In: L.E. Dias \& J.W.V. Mello. Recuperação de áreas degradadas. Viçosa, Universidade Federal de Viçosa.

Campos, H. 1979. Estatistica experimental não-paramétrica. Piracicaba, ESALQ.

Costa, R.C. \& Araújo, F.S. 2003. Densidade, germinação e flora do banco de sementes no solo, no final da estação seca, em uma área de caatinga, Quixadá, Ce.Acta Botanica Brasilica 17(2): 259-264.

Dalling, J.W.; Swaine, M.D. \& Garwood, N. 1998. Dispersal patterns and seed bank dynamics of pioneer trees in moist tropical forest. Ecology 79: 564-578.

Fenner, M. 1985. Seed ecology. London, Chapman and Hall.

Garwood, N.C. 1989. Tropical soil seed banks: a review. Pp. 148-209 In: M.A. Leck; T. Parker \& R.S. Simpson. Ecology soil seed banks. San Diego, Academic Press.

Hall, J.B. \& Swaine, M.B. 1980. Seed stocks in Ghanaian forest soil. Biotropica 12: 256-263.

Herrera, M.A.; Salamanca, C.P. \& Barea, J.M. 1993. Inoculation of woody legumes with selected arbuscular mycorrhizal fungi and rhizobia to recover desertified Mediterranean ecosystems. Applied Environmental Microbiology 59: 129-133.

Kageyama, P.Y. 1987. Conservação "In situ" de recursos genéticos de plantas. Revista IPEF 35: 7-35.

Martins, C.C. \& Silva, W.R. 1994. Estudos de bancos de sementes do solo. Informativo Abrates 4(1): 49-56.
Miriti, M.N. 1998. Regeneração florestal em pastagens abandonadas na Amazônia Central: competição, predação e dispersão de sementes. Pp. 179-190 In: C. Gascon \& P. Moutinho (eds.). Floresta amazônica: dinâmica, regeneração e manejo. Manaus, INPA.

Nepstad, D.C.; UHL, C.; Pereira, C.A. \& Silva, J.M.C. 1998. Estudos comparativo do estabelecimento de árvores em pastos abandonados e florestas adultas da Amazônia Oriental. Pp. 191-218. C. Gascon \& P. Moutinho (eds.). Floresta Amazônica: dinâmica, regeneração e manejo. Manaus, INPA.

Odum, E.P. 1988. Ecologia. Rio de Janeiro, Ed. Guanabara.

Oliveira Junior, R.S. \& Constantin, J. 2001.Plantasdaninhas, seu manejo. Guaíba, Agropecuária.

Sprent, J. \& Sutherland, J.M. 1990. Growth rates of three leguminous tree species on degraded acidic soils. Nitrogen Fixing The Research Reports 8: 17-23.

UHL, C.; Buschbacher, R. \& Serrão, E.A.S. 1988. Abandoned pastures in Eastern Amazonia. 1. Patterns of plants succession. Journal Ecology 76: 663-681.

UHL, C. \& Clarck, K. 1983. Seed ecology of selected Amazon basin successional species. Botanical Gazette 144: 419-425.

Viana, V.M. 1990. Seed and seedling availability as a basis for management of natural Forest regeneration. Pp. 99-115. In: A.B. Anderson. Alternatives to deforestation: steps toward sustainable use of the Amazon Rain Forest. New York, Columbia University Press.

Warr, S.J.; Thompson, K. \& Kent, M. 1993. Seed banks as a neglected area of biogeographic research: a review of literature and sampling techniques. Progress in Physical Geography 17: 329-347.

Whitmore, T.C. 1988. Forest dynamics and questions of scala. Pp. 13-17. In: M.E. Hadley. Rain forest Regeneration and Management. Paris, Int. Union of Biology Scence.

Whitmore, T.C. 1983. Secondary succession from seed in tropical rain forests. ForestryAbstracts 44(120): 767-779.

Whitmore, T.C. 1990. An introduction to tropical rain forest. Oxford, Clarendon Press.

Wiesenmüller, J.; Denich, M. \& Vleck, P.L.G. 1995. Regeneração vegetativa de capoeira na região de Amazônia Oriental, Brasil. Pp. 101-105. In: M. Kanashiro \& J.A. Parrota (eds.). Manejo e reabilitação de áreas degradadas e florestas secundárias na Amazônia. Belém, Embrapa.

Zar, J.H. 1996. Bioestatiscal analysis. New Jersey, PrenticeHall. 